This item was submitted to Loughborough's Research Repository by the author.

Items in Figshare are protected by copyright, with all rights reserved, unless otherwise indicated.

\title{
Measurement of instantaneous Achilles tendon moment arm and force during the stance phase of running
}

PLEASE CITE THE PUBLISHED VERSION

https://doi.org/10.1080/10255842.2021.1888938

\section{PUBLISHER}

Taylor and Francis

\section{VERSION}

AM (Accepted Manuscript)

\section{PUBLISHER STATEMENT}

This is an Accepted Manuscript of an article published by Taylor \& Francis in Computer Methods in Biomechanics and Biomedical Engineering on $22 \mathrm{Feb} 2021$, available online: http://www.tandfonline.com/10.1080/10255842.2021.1888938.

\section{LICENCE}

CC BY-NC-ND 4.0

\section{REPOSITORY RECORD}

Krikelis, Giorgos, Matthew Pain, and Laura-Anne Furlong. 2021. "Measurement of Instantaneous Achilles Tendon Moment Arm and Force During the Stance Phase of Running". Loughborough University. https://hdl.handle.net/2134/14223104.v1. 
1 Measurement of Instantaneous Achilles Tendon Moment Arm and Force during the

2

3

4

5

6 School of Sport, Exercise, and Health Sciences, Loughborough University, Loughborough,

7

LE11 3TU, United Kingdom

8

$9 \quad *$ Corresponding author telephone: +441509226440

10

*Corresponding author email: g.krikelis@,lboro.ac.uk

11 *Corresponding author address: School of Sport, Exercise, and Health Sciences,

12 Loughborough University, Loughborough, LE11 3TU, United Kingdom

13

\section{Stance Phase of Running}

Giorgos Krikelis*, Matthew T.G. Pain and Laura-Anne M. Furlong 


\section{Abstract}

2 Accurate estimates of the Achilles tendon (AT) moment arm (ATMA) are necessary for 3 investigating triceps surae muscle-tendon unit loading and function. There are limited reported

4 values of $\mathrm{AT}_{\mathrm{MA}}$ during running. By combining ultrasound and motion capture, $\mathrm{AT}_{\mathrm{MA}}$ was 5 estimated during the stance phase of running. Group mean $\mathrm{AT}_{\mathrm{MA}}$ was estimated at $49.2 \pm 3.8$

$6 \mathrm{~mm}$ and $37.5 \pm 5.3 \mathrm{~mm}$, relative to the centre of rotation (malleoli markers midpoint) and the

7 ankle finite helical axis respectively. Differences in the corresponding estimated AT forces

8 reached up to $3100 \mathrm{~N}$ approximately. Such discrepancies can lead to misinterpretation of the

9 whole muscle-tendon unit function.

11 Keywords: ultrasound, finite helical axis, tendon force, plantarflexors 


\section{Introduction}

Accurate estimates of the Achilles tendon (AT) moment arm (ATMA) are necessary for investigating triceps surae muscle-tendon unit loading, function, and computer simulation development. In general, the choice of moment arm measurement method can increase the variability in the reported moment arm lengths, both in patella (Tsaopoulos et al. 2006) and AT measurements (Sheenan 2012; Manal et al. 2013; Rasske et al. 2017; Wade et al. 2019). Ultrasound-based techniques that combine motion capture with ultrasound data have been used to acquire $\mathrm{AT}_{\mathrm{MA}}$ estimates during: rest, plantar-flexion contractions (Manal et al. 2013; Obst et al. 2017), controlled plantar-flexion tasks (Wade et al. 2019), and daily activities like walking and running (Farris et al. 2012a; Rasske et al. 2017). More recent studies have calculated $\mathrm{AT}_{\mathrm{MA}}$ relative to a finite helical axis (FHA) representation of the ankle axis of rotation (Hashizume et al. 2012; Sheehan 2012, Wade et al. 2019). There is evidence that the talocrural joint axis is not a fixed axis, changes substantially during ankle rotation, and shows significant variability between subjects (Lundberg et al. 1989). Invasive approaches show the ankle joint axis does not behave as a typical hinge joint and its orientation alters during locomotion (Arndt et al. 2004). Therefore, a joint axis that better describes the foot's rotation relative to the tibia, rather than the more conventional ankle joint axis or centre of rotation determined from the malleoli position, is required. The ankle FHA has been used for this purpose, facilitating more accurate $\mathrm{AT}_{\mathrm{MA}}$ estimates than can be obtained with MRI-based (Hashizume et al. 2012; Sheenan 2012) and motion capture/ ultrasound-based methods (Wade et al. 2019).

There are limited reported values of $\mathrm{AT}_{\mathrm{MA}}$ during running (Farris et al. 2012a), how it changes across typical ankle range of motion during stance phase and how it influences AT force, despite running being a common activity. The aim of this study was to estimate $\mathrm{AT}_{\mathrm{MA}}$ in typical healthy subjects during the stance phase of running by combining ultrasound and 
1 motion capture data. Specifically, to establish the difference in $\mathrm{AT}_{\mathrm{MA}}$ when calculated relative

2 to the malleoli midpoint (ankle centre of rotation) or relative to the ankle FHA, and their effects 3 on AT force estimates. 


\section{Subjects and Experimental Process}

Following Loughborough University ethics committee approval (SSEHS-2179), 12 healthy male subjects provided written informed consent to participate in this study (age: $28.6 \pm$ 5.9 years; mass: $78.9 \pm 9.4 \mathrm{~kg}$; height: $1.80 \pm 0.07 \mathrm{~m}$ ). Subjects were tested while running barefoot over a $13 \mathrm{~m}$ long lane, along which they had to accelerate, run at a steady velocity, and decelerate. For consistency, all subjects were asked to run using a forefoot strike. Ground reaction force and motion data were acquired from the steady state section with a mean velocity of $2.9 \pm 0.2 \mathrm{~m} / \mathrm{s}$. Prior to the running trials, twenty-nine $14 \mathrm{~mm}$ reflective markers were placed on the subject's dominant leg, waist and upper body (Figure 1), and tracked by 17 VICON Vantage cameras (250 Hz, VICON, Oxford Metrics Ltd., Oxford, UK). The calibrated motion capture volume was $2 \mathrm{~m} \mathrm{X} 3 \mathrm{~m} \mathrm{X} 3 \mathrm{~m}$, with all residual camera errors $<0.25 \mathrm{~mm}$, and most $<$ $0.15 \mathrm{~mm}$. A minimum of three successful trials per subject were captured, which was a single step with the preferred foot clearly landing on the force plate $(2000 \mathrm{~Hz}$, OR6, AMTI, Watertown, MA, USA).

An ultrasound probe was firmly strapped on the calf using an elastic bandage. The ultrasound system (Echoblaster LS128 CEXT-1Z; Telemed, Vilnius, Lithuania) with a 128element linear probe (LV7.5/60/128Z-2; B-mode; $8 \mathrm{MHz}$; $65 \mathrm{~mm}$ field of view; 65-80mm image depth) was placed on the medial gastrocnemius muscle tendon junction (MTJ). The ultrasound beamformer as well as the PC used to operate it were placed on a trolley which was run along next to the subject. Three reflective markers were placed non-collinearly on an L-shaped plastic cast that was rigidly wedged on the probe, without interfering with the scanning surface (Figure 1). These markers were used to calculate a local coordinate system on the probe, relative to which 
1 the orientation of the ultrasound image was defined. Images were collected at either 31 or 61 fps.

2 For each subject the dynamic range, focus, power and gain of the ultrasound were adjusted to optimise the image quality for digitising. This resulted in 8 of the 12 subjects having ultrasound images collected at $61 \mathrm{fps}$ and four at $31 \mathrm{fps}$. Motion analysis and ultrasound data were synchronised with a 5V TTL pulse emitted from the ultrasound beam former as soon as it started operating. The pulse was transmitted from an output port of the beam former, designed by the ultrasound manufacturer specifically for synchronisation purposes, to the motion capture system's analog-to-digital converter unit via a coaxial cable. The TTL pulse was sampled in VICON at $2000 \mathrm{~Hz}$ rate. The time difference between the first frame recorded in the ultrasound computer and the first data point of the TTL pulse recorded in VICON was measured and accounted for in order to correctly synchronise motion capture and ultrasound data.

On a separate day, unilateral lower limb MRI scans were acquired from the subjects' dominant limb. Axial spin-echo T1-weighted MRI scans (repetition time/echo time: 533845/7.546-16.940 ms; field of view: $240 \mathrm{~mm}$ x $240 \mathrm{~mm}$; slide thickness: $5 \mathrm{~mm}$; inter-slice spacing: $0 \mathrm{~mm}$ ) were acquired using a 3 Tesla scanner (Discovery MR750w, GE Healthcare, Buckinghamshire, UK). Subjects were positioned with their hips, knees and ankles secured in a neutral position (hip at $180^{\circ}$, knee at $180^{\circ}$, ankle at approximately $90^{\circ}$ ) using pads to minimise joint movement during scans.

\section{Data Analysis}

Marker and force data were filtered using a $4^{\text {th }}$ order digital Butterworth zero lag lowpass filter. The same cut-off frequency, $19 \mathrm{~Hz}$, was used to avoid impact artefacts (Kristianslund et al. 2012).

Estimation of the Ankle Finite Helical Axis 

segments. Initially, the rigid body transformations of the shank and foot segment were determined, following the procedures described in Challis (1995), in order to reduce marker position error due to soft tissue artefacts. A FHA was calculated based on the foot attitude matrices expressed in the shank coordinate system, (from frames) before and after a given

6 frame that corresponded to a minimum of $0.1 \mathrm{rad}$ foot rotation each (total rotation $0.2 \mathrm{rad}-$

$7 \quad$ Wade et al. 2019), using the methodology and the formulas from Spoor and Veldpaus (1980). However, to determine the orientation of the FHA, and the position of a point on the axis, the variation of the final equations described by Woltring et al. (1985) were used. A point and a unit vector of the 'mean' FHA $\left(\mathrm{FHA}_{\mathrm{m}}\right)$ were then calculated for each trial in the shank coordinate system $\left(\widehat{\boldsymbol{u}_{\boldsymbol{S C S}}}-\right.$ unit vector of the $\mathrm{FHA}_{\mathrm{m}}$ in the shank coordinate system), following the least-squares method of Lewis et al. (2006). The point and unit vector of the FHAm, expressed at the shank coordinate system at the initial calculation, were then transformed to the lab coordinate system $\left(\widehat{\boldsymbol{u}_{G C S}}-\right.$ unit vector of the $\mathrm{FHA}_{\mathrm{m}}$ in the lab or global coordinate system). The mean difference in position and orientation of the $\mathrm{FHA}_{\mathrm{m}}$ relative to bimalleolar axis was found for each subject and then for the whole group.

\section{Ultrasound Data and Achilles Tendon Moment Arm Calculation}

MTJ position was manually digitised on the ultrasound video frames by the same investigator, using a custom written MATLAB function. Following the calibration protocol described by Lichtwark and Wilson (2005) allowed digitised points from the ultrasound image to be projected to the lab coordinate system. Hence, the MTJ digitised coordinates were initially transformed to the ultrasound probe's local coordinate system, and then to the lab coordinate system. The vector formed by the MTJ position (expressed in the lab coordinate system) and the calcaneal marker, was used to define the line of action of the AT. 
2 ( $(\hat{\boldsymbol{l}})$ along its line of action and a position vector $(\overrightarrow{\boldsymbol{r}})$ from the ankle joint centre to any point 3 along the AT line of action (the position of the calcaneal marker was selected). In the case of a 4 centre of rotation (midpoint between malleoli markers), the magnitude of the $\mathrm{AT}_{\mathrm{MA}}$ was 5 calculated as the magnitude of the moment arm vector:

$$
A T_{M A}=|\vec{r} \times \hat{l}|
$$

7 In the case of a joint axis of rotation, the magnitude of the $\mathrm{AT}_{\mathrm{MA}}$ would be equal to the 8 projection of the moment arm vector to that axis of rotation (Zatsiorsky and Prilutsky 2012). 9 This is calculated as the scalar triple product:

$$
A T_{M A}=\left|\widehat{u_{G C S}} \cdot(\vec{r} \times \hat{l})\right|
$$

where $\overrightarrow{\boldsymbol{r}}$ in this case was the position vector from any point on the joint axis to any point along the AT line of action. The absolute value of the scalar product was used to ensure ATMA would be positive in case of a $\widehat{\boldsymbol{u}_{G C S}}$ change of direction.

Effect of MTJ Position Error on the Calculated ATMA and Calcaneus Insertion Position Correction

Various sources of error were involved in transforming the 2D MTJ position from the ultrasound image to the 3D lab coordinate system. Such errors originated from digitising, from potential movement of the markers attached on the ultrasound probe, from rotations of the probe relative to the skin, as well as from the accuracy of the calibration process. To estimate the digitising error, ultrasound images of the trials from one randomly selected subject were digitised repeatedly at three different occasions (up to 8 days post initial digitising) and the largest coefficient of variation that was calculated was assumed to be the digitising error. The error of projecting digitised points in the lab coordinate system was estimated by scanning a 
1 single grooved Perspex ${ }^{\circledR}$ sheet immersed into the water bath, following a similar procedure to

2 the one described in Lichtwark and Wilson (2005). The difference in the length of the vectors formed by the markers attached on the probe's cast was compared between static and running conditions and used to represent the error caused by marker movement. Finally, the probe's rotations relative to the skin were quantified using a marker cluster placed on the lower leg over the distal part of tibia (assuming that soft tissue movement would be lower in this area). The rotations were firstly measured during running in reference to a static pose, and then the effect of such rotations on the MTJ position as appearing in the ultrasound image was measured by rotating the probe manually (with the probe still attached on the subject's leg post-running). These errors were incorporated in the various steps needed to transform the MTJ coordinates from 2D (ultrasound image) to 3D (lab coordinate system) using error propagation formulas. This resulted in an error value for each of the MTJ coordinates (in 3D). The distance between the MTJ position and the MTJ position with the coordinate errors was assumed to be the total error of the 3D MTJ position.

To understand how the 3D MTJ position error propagated to the calculated $\mathrm{AT}_{\mathrm{MA}}$, its effect on the defined line of action of the AT had to be established. To achieve that, a pseudosensitivity analysis was done, where on a given trial, on every frame, the MTJ position was allowed to vary around its original position by a certain distance (the MTJ position error) in any possible direction. This could be visualised as a sphere of possible MTJ positions in the lab reference frame with the original MTJ position at its centre. Using spherical coordinates a new MTJ position was defined for combinations of azimuthal $\left(0 \leq \theta<2 \pi\right.$, increasing in $10^{\circ}$ intervals) and zenith angles ( $0 \leq \varphi<\pi$, increasing in $10^{\circ}$ intervals), using standard spherical to cartesian coordinate transformations. For every 'new' MTJ position, the line of action was redefined and the $\mathrm{AT}_{\mathrm{MA}}$ calculation was repeated. The root mean square difference was estimated 
1 at that frame. This was repeated for increasing radii (in $0.5 \mathrm{~mm}$ intervals) from $0.5 \mathrm{~mm}$ up to

2 the maximum value of the total estimated error of the MTJ position. This analysis was repeated

3 for one randomly selected trial from every participant, and the mean $\pm \mathrm{SD}$ and maximum $\mathrm{AT}_{\mathrm{MA}}$

4 error from all the frames of the trial was used to represent the $\mathrm{AT}_{\mathrm{MA}}$ error of the subject. From

5 the individual subject errors, the mean $\pm \mathrm{SD}$ and maximum $\mathrm{AT}_{\mathrm{MA}}$ error for the whole group

6 was estimated.

To acquire a better estimate of the calcaneus insertion position (CIP), the coordinates

\section{MRI Data and Cadaveric Regression Equation}

$\mathrm{AT}_{\mathrm{MA}}$ was measured from the MRI scans, using the 2D method described by Csapo et al. (2010), where a circle was fitted on the talus bone, and $\mathrm{AT}_{\mathrm{MA}}$ was calculated as the perpendicular line from the centre of the circle to the mid-line of the AT. This was repeated for eight different sagittal plane images where the talus bone and the AT were visible, and the mean $\mathrm{AT}_{\mathrm{MA}}$ was used for further analysis. Then, the regression equation of Klein et al. (1996)

(eq. 1), based on cadaver specimens, was used to estimate $\mathrm{AT}_{\mathrm{MA}}$ during the whole stance phase. The parameters of the equation were scaled to the MRI-derived ATMA measurement.

$$
A T_{M A}=51.068-1.166 * \theta_{\text {ankle }}
$$


1 In eq. $1, \operatorname{AT}_{\mathrm{MA}}(\mathrm{mm})$ is a function of ankle angle ( $\theta_{\text {ankle }}$ in radians), centred around $90^{\circ}$ ankle

2 angle, with positive and negative denoting dorsi- and plantar-flexion of the ankle joint. To scale eq. 1, the constant indicating where the curve intercepts the y-axis (51.068) was replaced with

4 5 the MRI-derived $\mathrm{AT}_{\mathrm{MA}}$ value, while their difference expressed relative to the original value,

$$
\text { scale }=\frac{M R I_{M A}-51.068}{51.068}
$$

was used to scale the gradient proportionally:

$$
\text { gradient }_{\text {scaled }}=-1.166-\text { scale } * 1.166
$$

The mean ankle dorsi-/ plantar-flexion angle range across the stance phases of the three trials of each subject, was used to acquire an estimate of $\mathrm{AT}_{\mathrm{MA}}$ across stance phase.

\section{Inverse Dynamics and Achilles Tendon Force Estimation}

Using the filtered data, ankle plantar-/ dorsi-flexion moment during stance phase was calculated with standard inverse dynamics, using a custom written MATLAB function (R2017a; The MathWorks, Inc., Natick, MA, USA). Ankle moment was calculated about the malleoli midpoint in the foot coordinate system. Then, a new foot coordinate system was defined with one axis aligned with $\widehat{\boldsymbol{u}_{G C S}}$, and the ankle moment was recalculated about a point on that axis. For consistency, the point along this axis (aligned with $\widehat{\boldsymbol{u}_{G C S}}$ ) that had shortest distance from the malleoli midpoint was chosen every time. Inertial parameters of each segment were calculated based on body mass and segment lengths, and the methods from de Leva (1996).

AT force was calculated during stance phase by dividing the ankle plantar-flexion moment by the instantaneous $\mathrm{AT}_{\mathrm{MA}}$. This was done for each trial of every subject. First, AT force was estimated using the ankle moment and $\mathrm{AT}_{\mathrm{MA}}$ calculated relative to the $\mathrm{FHA}_{\mathrm{m}}$. Then, 
1 AT force was also estimated by dividing the ankle moment about the malleoli midpoint with

2 both the $\mathrm{AT}_{\mathrm{MA}}$ calculated relative to that same point and the MRI-based $\mathrm{AT}_{\mathrm{MA}}$.

\section{$3 \quad$ Statistical analysis}

The subject mean $\mathrm{AT}_{\mathrm{MA}}$ curve and AT force curves were calculated across all subject's

5 trials, and the group mean $\mathrm{AT}_{\mathrm{MA}}$ and $\mathrm{AT}$ force was calculated across all subjects (the mean of

6 the subject means), for every method used. AT $\mathrm{TA}_{\mathrm{MA}}$ and AT force from each trial were interpolated

7 to 51 points across stance phase using cubic spline interpolation. Root mean square difference

8 (RMSD) across stance was calculated between the two ultrasound-based methods. A $2^{\text {nd }}$ degree

9 polynomial was fitted on each subject's mean $\mathrm{AT}_{\mathrm{MA}}$ curve (mean of subject's trials) against normalised foot contact time. Statistical Parametric Mapping (SPM) was used on individual $\mathrm{AT}_{\mathrm{MA}}$ and AT force curves to determine significant differences, with an alpha level of $\leq 0.05$. All SPM analyses were implemented using the open-source spm1d code (v.M0.1, www.spm1d.org) in MATLAB. The scalar output statistic, $\operatorname{SPM}\{\mathrm{F}\}$, was calculated separately at each individual time node and referred to as a SPM. A critical threshold, where only $5 \%$ of smooth random curves were expected to traverse, was calculated based on estimates of trajectory smoothness via temporal gradients (Friston et al. 2007). The null hypothesis tested was differences in $\mathrm{AT}_{\mathrm{MA}}$ and $\mathrm{AT}$ force at each instant in time across the stance were not affected by the method used to elicit ATMA. 
For all trials from all subjects the centre of pressure at foot strike was located at over $65 \%$ along the line that originates from the heel marker and finishes at the toe marker. Digitising, ultrasound calibration, probe rotations and marker movement error resulted in $4.2 \pm 0.6 \mathrm{~mm}$ (maximum $7.4 \mathrm{~mm}$ ) error in the MTJ position when transformed into the lab coordinate system. Results from the pseudo-sensitivity analysis showed that the $\mathrm{AT}_{\mathrm{MA}}$ error due to the maximum MTJ position error was estimated at $0.1 \pm 0.1 \mathrm{~mm}$ (maximum $0.2 \mathrm{~mm}$ ), which is below the resolution of the motion capture system. The change in position and orientation of the $\mathrm{FHA}_{\mathrm{m}}$ relative to the malleoli axis can be seen in Figure 2. When combining motion capture with ultrasound data group mean $\mathrm{AT}_{\mathrm{MA}}$ was estimated at $49.2 \pm 3.8 \mathrm{~mm}$ and $37.5 \pm 5.3 \mathrm{~mm}$, relative to the ankle centre of rotation (malleoli markers midpoint) and the $\mathrm{FHA}_{\mathrm{m}}$ respectively. The RMSD between these two ultrasound-based methods across stance phase was estimated at 12.1 $\pm 6.4 \mathrm{~mm}$. The parameters of the $2^{\text {nd }}$ degree polynomial fitted on the ultrasound-based $\mathrm{AT}_{\mathrm{MA}}$ and their corresponding $\mathrm{R}^{2}$ can be seen in Table 1 . There was very good agreement between the fitted polynomials and the measured $\mathrm{AT}_{\mathrm{MA}}$. The individual subject curves and the subject variation for both ultrasound-based $\mathrm{AT}_{\mathrm{MA}}$ can be seen in Figure 3. SPM analysis revealed statistically significant differences in $\mathrm{AT}_{\mathrm{MA}}$ from different methods across the entire stance phase $(\mathrm{p}<0.001)$. The scaled regression equation from Klein et al. (1996) resulted in group mean $\mathrm{AT}_{\mathrm{MA}}$ of $55.2 \pm$ $3.0 \mathrm{~mm}$, while the $\mathrm{AT}_{\mathrm{MA}}$ curve profile appeared 'flatter' (Figure 4), in comparison to the motion capture/ ultrasound derived $\mathrm{AT}_{\mathrm{MA}}$. The RMSD between the regression equation $\mathrm{AT}_{\mathrm{MA}}$ and the $\mathrm{AT}_{\mathrm{MA}}$ relative to the $\mathrm{FHA}_{\mathrm{m}}$ across stance phase was estimated at $18.5 \pm 6.5 \mathrm{~mm}$.

Ankle moment calculated relative to a point on the $\mathrm{FHA}_{\mathrm{m}}$ was greater in magnitude than when calculated relative to the malleoli midpoint, with a RMSD of $12.1 \pm 5.4 \mathrm{~N} \mathrm{~m}$ (Figure 5). The RMSD in AT force across stance phase was $1456 \pm 898 \mathrm{~N}$ between the two ultrasoundbased methods, and $1965 \pm 955 \mathrm{~N}$ between using the ultrasound/ $\mathrm{FHA}_{\mathrm{m}}$ based and regression 
1 equation based $\mathrm{AT}_{\mathrm{MA}}$. Group mean peak $\mathrm{AT}$ force when using the $\mathrm{AT}_{\mathrm{MA}}$ relative to the $\mathrm{FHA}_{\mathrm{m}}$, $27192 \pm 1637 \mathrm{~N}$, was higher than both the $\mathrm{AT}_{\mathrm{MA}}$ relative to the malleoli midpoint and from the

3 scaled regression equation (Figure 5). Absolute differences in peak AT force reached $2249 \mathrm{~N}$ 4 and $3103 \mathrm{~N}$, respectively. SPM analysis revealed statistically significant differences in AT force 5 from different methods across the entire stance phase $(\mathrm{p}<0.001)$.

6

7

8

9

10

11

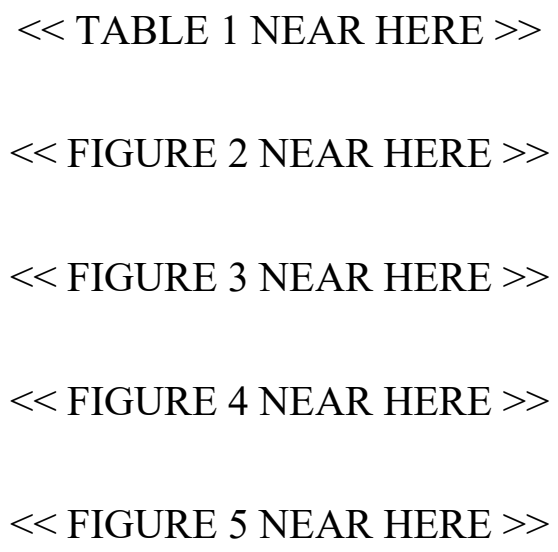


In the current study, $\mathrm{AT}_{\mathrm{MA}}$ was measured in vivo during running by combining motion capture and ultrasound data. Group mean $\mathrm{AT}_{\mathrm{MA}}$ was estimated at $49.2 \pm 3.8 \mathrm{~mm}$ and $37.5 \pm 5.3$ $\mathrm{mm}$, when calculated relative to the centre of rotation (malleoli markers midpoint) and relative to the ankle axis of rotation $\left(\mathrm{FHA}_{\mathrm{m}}\right)$ respectively. $\mathrm{AT}_{\mathrm{MA}}$ estimated from different methods were significantly different across the entire stance phase. The impact of these differences in $\mathrm{AT}_{\mathrm{MA}}$ values between methods was highlighted when AT force was estimated, with differences in peak AT force reaching up to 3100 N. Such discrepancies can not only change the estimated load that AT undergoes, but also misinterpret the function and the demand in force production from the triceps surae.

The $\mathrm{AT}_{\mathrm{MA}}$ curve across the ankle range of motion from the motion analysis/ ultrasound data relative to the $\mathrm{FHA}_{\mathrm{m}}$ intersected with the $\mathrm{AT}_{\mathrm{MA}}$ profile during walking reported by Rasske et al. (2017) that was measured with a similar method (Figure 6). However, in that study the moment arm was measured relative to the midpoint of the malleoli markers. In contrast to the results from Wade et al. (2019), $\mathrm{AT}_{\mathrm{MA}}$ relative to the ankle 'functional' axis were lower than when calculated relative to the malleoli midpoint in this study. The reasons for such difference are not clear, since the methods used in this study and in Wade et al. (2019) were similar. Part of it could be explained by the fact that the loading conditions were different (running vs. toe raises), as well as subject anatomical variation.

\section{$<<$ FIGURE 6 NEAR HERE $>>$}

The decrease in $\mathrm{AT}_{\mathrm{MA}}$ when calculated relative to the $\mathrm{FHA}_{\mathrm{m}}$ as opposed to the centre of rotation (malleoli midpoint) can be explained primarily by the different position and orientation of the $\mathrm{FHA}_{\mathrm{m}}$ relative to the bimalleolar axis. Previous invasive and radiostereometric analysis studies have shown substantial deviation (Lundberg et al. 1989; Arndt et al. 2004) of the ankle 
1 FHA relative to the bimalleolar axis. Such deviation was also observed in this study (Figure 2)

2 and would bring the axis of rotation closer to the AT line of action, explaining the $\mathrm{AT}_{\mathrm{MA}}$ decrease when $\mathrm{FHA}_{\mathrm{m}}$ was used compared to the malleoli midpoint. The $\mathrm{FHA}_{\mathrm{m}}$ difference in orientation and position compared to the bimalleolar axis varied between subjects (Figure 2), contributing to the variation of the individual $\mathrm{AT}_{\mathrm{MA}}$ curves (Figure 3). Finally, an observation worth noting was about the characteristic 'step' of the $\mathrm{FHA}_{\mathrm{m}}$ based $\mathrm{AT}_{\mathrm{MA}}$ curve profile on both the group mean and individual curves, at approximately 20\% of stance phase (Figure 3 and Figure 4A). Although this step can be seen on some individual curves on the malleoli midpoint ATMA (Figure 3), it is certainly much more prominent on the $\mathrm{FHA}_{\mathrm{m}}$ based $\mathrm{AT}_{\mathrm{MA}}$. This raises the question whether this step is an actual physiological change of the ATMA or simply an artefact of the ultrasound-based methods. In either case it seems that the curve step is better observed (or amplified if it is an artefact) when using the $\mathrm{FHA}_{\mathrm{m}}$ based $\mathrm{AT}_{\mathrm{MA}}$. Also, considering that a polynomial was fitted on these curves and despite the goodness of the fit (Table 1), a similar question can be asked on whether the smoothness of a $2^{\text {nd }}$ degree polynomial that would erase that curve step is justified (if it was an artefact) or a higher order would be more appropriate to better describe that subtle $\mathrm{AT}_{\mathrm{MA}}$ change. Based on the results from this study it would be challenging to determine whether it is a method artefact, and hence which order polynomial would be more appropriate. Future work would be required to categorically determine that.

The $\mathrm{FHA}_{\mathrm{m}}$ based $\mathrm{AT}_{\mathrm{MA}}$, when compared to the values from the literature on Figure 6, and to similar figures from other papers (Donnelly et al. 2019; Rasske et al. 2017; Sheenan 2012), seemed to lie closer to the results of Wade et al. (2019) (results relative to the malleoli midpoint), Rasske et al. (2017), Hashizume et al. (2012), and Manal et al. (2013). From these studies only Hashizume et al. (2012) also calculated the ankle FHA, measuring $\mathrm{AT}_{\mathrm{MA}}$ at rest with MRI. It could be argued that the results from this study, as well as the ones from Hashizume et al. (2012), seemed to be on the lower end of the reported ATMA relative to the ankle's FHA. 
1 The increase of moment arm magnitude at greater muscle efforts has been observed repeatedly

2 (Maganaris 2004; Hashizume et al. 2014), but assessing the level of effort exerted during running in this study relative to 'loaded' conditions from other studies was not possible. In line with previous findings, the motion capture/ ultrasound derived $\mathrm{AT}_{\mathrm{MA}}$ measured in this study increased during plantar-flexion as shown in Figure 4B (Fath et al. 2010; Hashizume et al. 2012; Wade et al. 2019). However, it should be noted that other research, including MRI-based/ FHA studies, have not shown a consistent trend in $\mathrm{AT}_{\mathrm{MA}}$ change with ankle angle at various contraction levels (Hashizume et al. 2012; Sheenan 2012; Manal et al. 2013; Olszewski et al. 2015).

Differences in $\mathrm{AT}_{\mathrm{MA}}$ from different methods were statistically significant across the entire stance phase. Group mean $\mathrm{AT}_{\mathrm{MA}}$ from Klein et al. (1996) regression was $49 \%$ higher than the ultrasound derived $\mathrm{FHA}_{\mathrm{m}}$ based $\mathrm{AT}_{\mathrm{MA}}$. In general, 2D-MRI has been reported previously to overestimate $\mathrm{AT}_{\mathrm{MA}}$ (up to $11 \mathrm{~mm}$ Hashizume et al. 2012; up to $3 \mathrm{~mm}$, Sheenan 2012). The $\mathrm{AT}_{\mathrm{MA}}$ derived from the Klein et al. (1996) regression equation was scaled to the moment arm measured from the MRI scans in 2D and this could explain the $\mathrm{AT}_{\mathrm{MA}}$ offset compared to the ultrasound/ motion capture-based method. As a general comment, $\mathrm{AT}_{\mathrm{MA}}$ from the scaled regression equation showed an improbably low intra-subject variability, something that is likely inherent to using a regression equation with a single independent variable (ankle dorsi-/ plantar-flexion angle). Finally, the lack of a more prominent $\mathrm{AT}_{\mathrm{MA}}$ change with ankle angle highlights another limitation of this approach.

A primary application for $\mathrm{AT}_{\mathrm{MA}}$ is to assess the load that AT undergoes during locomotion. Therefore, it would be useful to determine the difference in the estimated AT force from the different methods of acquiring the $\mathrm{AT}_{\mathrm{MA}}$. Peak AT force from the ultrasound/ motion capture with FHAm method reached $7192 \mathrm{~N}$, and was $2249 \mathrm{~N}$ and $3103 \mathrm{~N}$ higher than from the ultrasound/ motion capture with centre of rotation (malleoli midpoint) and from the regression equation. Using the group mean weight $(774 \mathrm{~N})$, these differences correspond to 2.9 and 4.0 
1 bodyweights. Such substantial discrepancies in peak AT forces would indicate differences in the

2 loading rate of the tendon, in the force demand from the lower extremity musculature, and potentially in the overall function of the whole muscle-tendon unit. Disregarding how robust each method is, as well as the amount of anatomical and kinematic information each method uses to estimate $\mathrm{AT}_{\mathrm{MA}}$, it would be hard to assess which forces correctly represent the load of the AT during running. However, an assumption (and limitation) when estimating AT force from the externally measured plantar-flexion moment, is that the contribution of the AT force to the plantar-flexion moment is assumed to be $100 \%$. In previous in vivo invasive studies, the contribution of the AT force in the plantar-flexion moment has been estimated at approximately $65-67.4 \%$ (Arndt et al. 1998; Gregor et al. 1991). Hence, 65\% of that estimated $7192 \mathrm{~N}$ force would result in $4675 \mathrm{~N}$. Considering that peak AT forces up to $5000 \mathrm{~N}$ have been directly measured in humans during running at a similar running speed $\left(\sim 3 \mathrm{~m} \cdot \mathrm{s}^{-1}-\right.$ Komi 1990), the estimated AT forces in the current study when using the motion capture/ ultrasound derived FHA $_{\mathrm{m}}$ based seem plausible in general. In comparison, $65 \%$ of peak forces from the malleoli midpoint and regression equation (MRI) based $\mathrm{AT}_{\mathrm{MA}}$, would result in less than $3300 \mathrm{~N}$ and 2700 $\mathrm{N}$ peak AT force, less than what has been measured in vivo at a similar speed.

Certain assumptions and limitations inherent to the overall approach that was followed in this study should be mentioned. Firstly, the 2D-MRI method (or considering the midpoint of the malleoli markers as the ankle centre of rotation) neglect any motion of the talus bone, which along with the tibia and fibula constitutes the ankle joint. At the same time, any 2D approach automatically follows the assumption that the ankle joint axis is oriented perpendicularly to the sagittal plane. While this is avoided when calculating the ankle's FHA, every FHA would have to be derived not only from the rotation of the talus about the tibia, but also of the other (smaller) bones about the tibia. Future research could focus on combining the MRI measurements with the motion capture/ ultrasound data to locate the position of specific 
1 anatomical landmarks such as the talus bone. A second limitation of this approach is that the current 2D (or pseudo-3D) ultrasound-based method can be used to describe AT kinematics during normative ankle joint rotation and triceps surae function and architecture. In such joints (and muscle-tendon units), it is a more reasonable assumption that the ankle plantar-/ dorsi5 flexion takes place primarily on one plane and emanates from the triceps surae muscle contraction. This automatically renders the use of this method problematic when it comes to measuring the $\mathrm{AT}_{\mathrm{MA}}$ during pathological gait. Finally, defining the $\mathrm{AT}$ as a line between the calcaneus insertion and the MTJ, assumes that the AT is being deformed only longitudinally. Research findings have shown that plantar-flexion contractions can cause transverse as well as the longitudinal deformation of the AT (Farris et al. 2012b). Such transverse deformation cannot be taken into account using the current method.

Although some limitations of the methods used in this study have already been discussed, some additional technical limitations need to be acknowledged. Soft tissue artefacts rigid body have certainly influenced the FHA calculations, which is known to be sensitive to noisy data (Woltring et al. 1985). Indeed, the relative movement between the smaller bones of the foot is substantial during the stance phase of running, causing substantial rotations about the joints in the foot (Arndt et al., 2007). In addition, the limited range of motion of the ankle (compared to more mobile joints, such as the hip or knee) would be an inherent limitation when attempting to calculate the ankle's FHA. Furthermore, calculating the single FHA $_{m}$ would certainly introduce some error, as the orientation of the ankle joint axis has been shown to change during different types of rotation (dorsi-/ vs. plantar-flexion) as well as during rotations at different anatomical planes (Lundberg et al. 1989). Regarding the tracking of the MTJ, the amount of movement outside the plane of the ultrasound image plane could not be estimated. This is inevitable when measuring the movement of a three-dimensional object (MTJ) with 
1 two-dimension imaging equipment (ultrasound). Furthermore, the MTJ approach of creating

2 the AT vector could potentially 'drag' its line of action posteriorly of the shank, potentially

3 leading to an $\mathrm{AT}_{\mathrm{MA}}$ overestimation. However, it was estimated that moving the MTJ position

4 up to $7.4 \mathrm{~mm}$ away from its position had a negligible effect, causing less than $0.5 \mathrm{~mm} \mathrm{AT} \mathrm{MA}$

5 change. In comparison, previous studies have mounted the probe lower on the shank where the

6 free part of the AT could be visualised (Rasske et al. 2017; Wade et al. 2019). This was found

7 practically challenging, as the probe movement relative to the skin increased substantially

8 during running. Finally, it should be noted that based on pilot work done prior to this study and

9 with the current means available, it was found that above approximately $4 \mathrm{~m} \cdot \mathrm{s}^{-1}$ running

10 velocities, the probe and soft tissue motion (and hence, the ultrasound image artefacts) would increase substantially. This could only be constrained with excessively tight bandaging that could potentially affect gastrocnemius function and surely the ultrasound image.

In this study, $\mathrm{AT}_{\mathrm{MA}}$ was calculated during running based on a combination of motion capture and ultrasound measurements. The $\mathrm{AT}_{\mathrm{MA}}$ was found to be smaller when measured relative to the ankle's FHA compared to the common ankle joint centre definition, the malleoli markers midpoint. This resulted in peak AT force estimates being $2249 \mathrm{~N}$ higher when using the ankle's FHA compared to the malleoli midpoint. The estimated peak AT forces when using the $\mathrm{AT}_{\mathrm{MA}}$ relative to the ankle's FHA were similar to AT forces that were measured directly on the tendon in past invasive research, supporting the relevance of the $\mathrm{AT}_{\mathrm{MA}}$ values calculated here with the FHA method. 


\section{Disclosure Statement}

2 No potential conflict of interest was reported by the authors.

3 


\section{$1 \quad$ References}

2 Arndt AN, Komi PV, Bruggemann GP, Lukkariniemi J. 1998. Individual muscle contributions 3 to the in vivo Achilles tendon force. Clin Biomech. 13(7):532-541.

4 Arndt AN, Westblad P, Winson I, Hashimoto T, Lundberg A. 2004. Ankle and subtalar 5 kinematics measured with intracortical pins during the stance phase of walking. Foot Ankle Int. $6 \quad 25(5): 357-364$.

7 Arndt AN, Wolf P, Liu A, Nester C, Stacoff A, Jones R, Lundgren P and Lundberg A. 2007. 8 Intrinsic foot kinematics measured in vivo during the stance phase of slow running. J Biomech. $9 \quad 40: 2672-2678$.

Challis JH. 1995. A procedure for determining rigid body transformation parameters. J Biomech. 28(6):733-737.

12 Csapo R, Maganaris CN, Seynnes OR, Narici MV. 2010. On muscle, tendon and high heels. J 13 Exp Biol. 213:2582-2588.

14 de Leva P. 1996. Adjustments to Zatsiorsky-Seluyanov's segment inertia parameters. J 15 Biomech. 29(9):1223-1230.

Donnelly CJ, Alexander CF, Stannage K, Reid S. 2019. A commentary on Kalkman et al.'s

17 letter to the editor regarding Alexander et al., 2019: "Children with cerebral palsy have larger 18 in-vivo and linearly scaled Achilles tendon moment arms than typically developing children".

19 J. Biomech. 92:178-180. Farris DJ, Buckeridge E, Trewartha G, McGuigan MP. 2012a. The effect of orthotic heel lifts on Achilles tendon strain during walking and running. J Appl Biomech. 28(5):511-519. 
1 Farris DJ, Trewartha G, McGuigan MP, Lichtwark GA. 2012b. Differential strain patterns of the

2 human Achilles tendon determined in vivo with freehand three-dimensional ultrasound imaging.

$3 \quad$ J Exp Biol. 216:594-600.

4 Fath F, Blazevich AJ, Waugh CM, Miller SC, Korff T. 2010. Direct comparison of in vivo 5 Achilles tendon moment arms obtained from ultrasound and MR scans. J Appl Biomech. 6 109(6):1644-1652.

7 Friston K, Ashburner J, Kiebel S, Nichols T, Penny W. 2007. Statistical Parameter Mapping:

8 The Analysis of Functional Brain Images. London (UK): Elsevier.

9 Gregor RJ, Komi PV, Browning RC, Jarvinen M. 1991. A comparison of the triceps surae and residual muscle moments at the ankle during cycling. J Biomech. 24(5):287-297. determination of the Achilles tendon moment arm in three-dimensions. J Biomech. 45(2):409413.

Hashizume S, Iwanuma S, Akagi R, Kanehisa H, Kawakami Y, Yanai T. 2014. The contractioninduced increase in Achilles tendon moment arm: A three-dimensional study. J Biomech. 47(12):3226-3231.

Klein P, Mattys S, Rooze M. 1996. Moment arm length variations of selected muscles acting on talocrural and subtalar joints during movement: An in vitro study. J Biomech. 29(1):21-30.

19 Komi PV. 1990. Relevance of in vivo force measurements to human biomechanics. J Biomech. 23 Suppl 1:23-34.

Kristianslund E, Krosshaug T, van den Bogert AJ. 2012. Effect of low pass filtering on joint moments from inverse dynamics: Implications for injury prevention. J Biomech. 45(4):666-671. 
1 Lewis GS, Sommer HJ, Piazza SJ. 2006. In vitro assessment of a motion-based optimization

2 method for locating the talocrural and subtalar joint axes. J Biomech Eng. 128(4):596-603.

3 Lichtwark GA, Wilson AM. 2005. In vivo mechanical properties of the human Achilles tendon

4 during one-legged hopping. J Exp Biol. 208:4715-4725.

5 Lundberg A, Svensson OK, Nemeth G, Selvik G. 1989. The axis of rotation of the ankle joint. J

$6 \quad$ Bone Joint Surg Br. 71(1): 94-99.

7 Maganaris CN. 2004. Imaging-based estimates of moment arm length in intact human muscle8 tendons. Eur J Appl Physiol. 91(2-3):130-139.

9 Manal K, Cowder JD, Buchanan TS. 2013. Subject-specific measures of Achilles tendon 10 moment arm using ultrasound and video-based motion capture. Physiol Rep. 1(6):e00139.

11 Obst SJ, Barber L, Miller A, Barrett RS. 2017. Reliability of Achilles tendon moment arm measured in vivo using freehand three-dimensional ultrasound. J Appl Biomech. 33(4):300-304.

Olszewski K, Dick TJM, Wakeling JM. 2015. Achilles tendon moment arms: The importance of measuring at constant tendon load when using the tendon excursion method. J Biomech. 48(6):1206-1209.

Rasske K, Thelen DG, Franz JR. 2017. Variation in the human Achilles tendon moment arm during walking. Comput Methods Biomech Biomed Engin. 20(2):201-205.

Sheenan FT. 2012. The 3D in vivo Achilles' tendon moment arm, quantified during active muscle control and compared across sexes. J Biomech. 45(2):225-230.

Spoor CW, Veldpaus FE. 1980. Rigid body motion calculated from spatial co-ordinates of markers. J Biomech. 13(4):391-393. 
1 Tsaopoulos DE, Baltzopoulos V, Maganaris CN. 2006. Human patellar tendon moment arm

2 length: Measurement considerations and clinical implications for joint loading assessment. Clin 3 Biomech. 21(7):657-667.

4 Wade FE, Lewis GS, Piazza SJ. 2019. Estimates of Achilles tendon moment arm differ when 5 axis of ankle rotation is derived from ankle motion. J Biomech. 90:71-77.

6 Woltring HJ, Huiskes R, de Lange A, Veldpaus FE. 1985. Finite centroid and helical axis 7 estimation from noisy landmark measurements in the study of human joint kinematics. J 8 Biomech. 18(5):379-389.

9 Zatsiorsky VM, Prilutsky BI. 2012. Biomechanics of Skeletal Muscles. Leeds (UK): Human $10 \quad$ Kinetics. p. 269. 


\section{List of Tables}

Table 1. Presented results per subject are: the parameters and $\mathrm{R}^{2}$ of the $2^{\text {nd }}$ degree polynomials that were fitted on the subject mean $A T_{M A}$ curves (mean of subject's trials) against the normalised foot contact time, from the two ultrasound based methods. The $2^{\text {nd }}$ degree polynomial is of the following format, $A T_{M A}=a t^{2}+b t+c$, with $t$ the normalised foot contact time taking values in the $[0,1]$ range. Also, the subject mean $\pm \mathrm{SD}_{\text {minimum }} \mathrm{AT}_{\mathrm{MA}}$ across stance phase from the ultrasound-based methods, as well as from the regression equation from Klein et al. (1996) that was scaled to the ATMA measured in the MRI scans.

\begin{tabular}{|c|c|c|c|c|c|c|c|c|c|c|c|}
\hline & \multicolumn{5}{|c|}{ Ankle axis of rotation (FHAm) } & \multicolumn{5}{|c|}{ Ankle axis of rotation (centre of rotation) } & \multirow{2}{*}{$\begin{array}{c}\frac{\text { Regression equation, }}{\text { scaled to MRI* }} \\
\text { Minimum AT MA (mm) }\end{array}$} \\
\hline & $\mathbf{a}$ & b & c & $\mathbf{R}^{\mathbf{2}}$ & $\begin{array}{c}\text { Minimum } \\
\text { AT }_{\text {ma }}(\mathbf{m m})\end{array}$ & $\mathbf{a}$ & b & c & $\mathbf{R}^{2}$ & $\begin{array}{c}\text { Minimum } \\
\text { AT }_{\text {MA }}(\mathbf{m m}) \\
\end{array}$ & \\
\hline P01 & 0.0449 & -0.0384 & 0.0444 & 0.98 & $36.3 \pm 4.7$ & 0.0377 & -0.0327 & 0.0588 & 0.99 & $51.9 \pm 0.8$ & $58.1 \pm 0.1$ \\
\hline P02 & 0.0061 & -0.0071 & 0.0351 & 0.75 & $32.9 \pm 0.2$ & 0.0134 & -0.0135 & 0.0525 & 0.96 & $49.1 \pm 0.5$ & $52.7 \pm 0.0$ \\
\hline P03 & 0.0284 & -0.0219 & 0.0258 & 0.96 & $21.5 \pm 2.5$ & 0.0294 & -0.0239 & 0.0541 & 0.94 & $48.7 \pm 0.6$ & $58.1 \pm 0.0$ \\
\hline P04 & 0.0538 & -0.0521 & 0.0466 & 0.95 & $33.4 \pm 0.5$ & 0.0418 & -0.0409 & 0.0588 & 0.99 & $48.7 \pm 1.0$ & $56.5 \pm 0.0$ \\
\hline P05 & 0.0434 & -0.0403 & 0.0458 & 0.99 & $36.3 \pm 1.2$ & 0.0474 & -0.0431 & 0.0548 & 0.99 & $44.9 \pm 0.8$ & $50.7 \pm 0.0$ \\
\hline P06 & 0.0517 & -0.0491 & 0.0445 & 0.93 & $33.3 \pm 2.6$ & 0.0510 & -0.0471 & 0.0544 & 0.95 & $44.0 \pm 0.3$ & $53.3 \pm 0.0$ \\
\hline P07 & 0.0304 & -0.0263 & 0.0441 & 0.90 & $38.2 \pm 0.6$ & 0.0347 & -0.0309 & 0.0536 & 0.99 & $46.9 \pm 0.3$ & $52.7 \pm 0.0$ \\
\hline P08 & 0.0336 & -0.0301 & 0.0399 & 0.99 & $32.9 \pm 0.9$ & 0.0283 & -0.0260 & 0.0466 & 0.99 & $40.5 \pm 0.5$ & $50.5 \pm 0.0$ \\
\hline P09 & 0.0564 & -0.0522 & 0.0527 & 0.98 & $40.1 \pm 0.9$ & 0.0460 & -0.0416 & 0.0589 & 0.99 & $49.2 \pm 0.7$ & $59.3 \pm 0.0$ \\
\hline P10 & 0.0491 & -0.0447 & 0.0446 & 0.98 & $34.0 \pm 1.3$ & 0.0491 & -0.0458 & 0.0537 & 0.99 & $42.8 \pm 0.6$ & $53.9 \pm 0.0$ \\
\hline P11 & 0.0541 & -0.0497 & 0.0467 & 0.99 & $35.0 \pm 1.6$ & 0.0464 & -0.0417 & 0.0502 & 0.99 & $40.4 \pm 0.9$ & $56.7 \pm 0.0$ \\
\hline P12 & 0.0416 & -0.0359 & 0.0372 & 0.96 & $28.9 \pm 0.6$ & 0.0279 & -0.0240 & 0.0469 & 0.95 & $41.6 \pm 0.3$ & $56.0 \pm 0.0$ \\
\hline
\end{tabular}

$* \pm 0.0$ indicates standard deviation less than $0.1 \mathrm{~mm}$ 
List of Figures

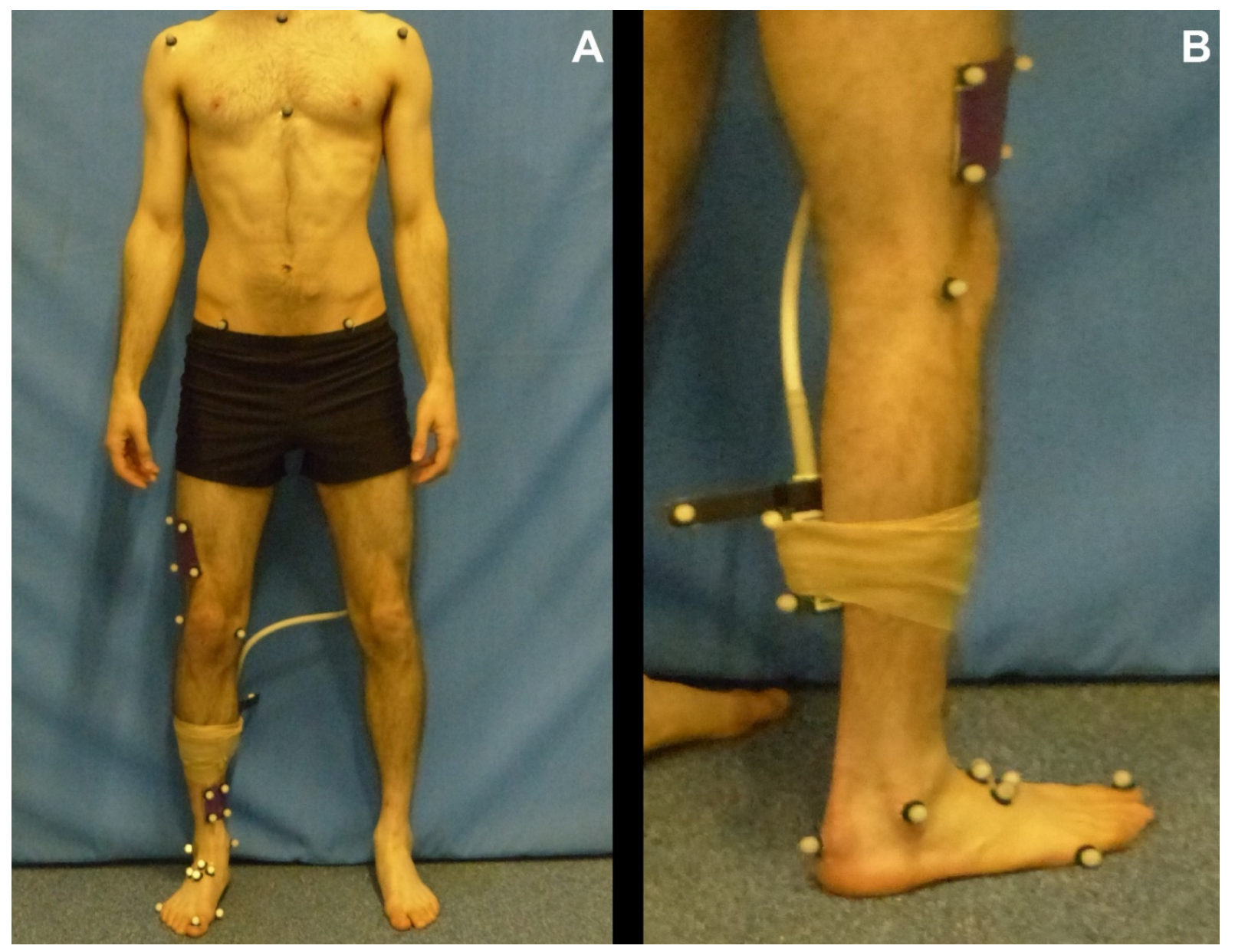

Figure 1. Marker placements. 


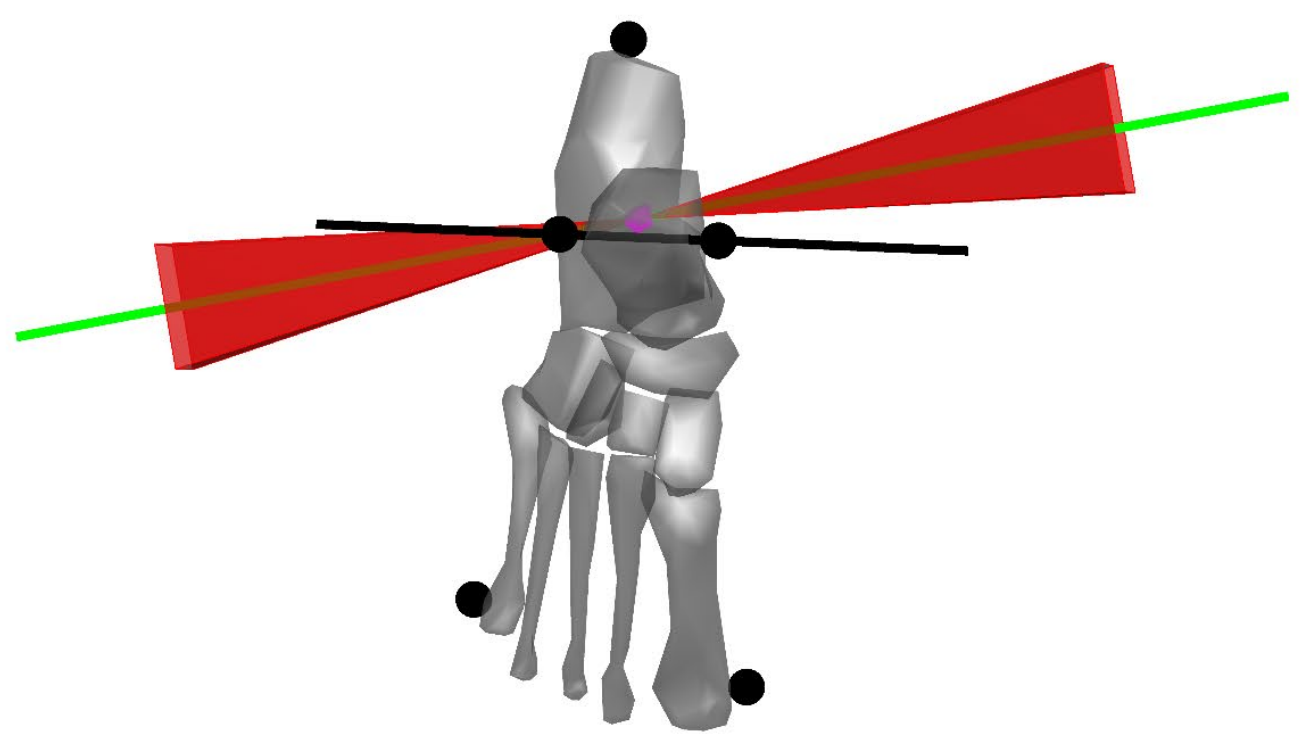

Figure 2. The position and orientation of the group mean ankle FHA (green) relative to the bimalleolar axis (black). The red cone surfaces represent the SD of the $\mathrm{FHA}_{\mathrm{m}}$ orientation, while the magenta cluster represents the $95 \%$ confidence intervals of the points that the FHA $\mathrm{m}$ could travel through. It should be noted that the relative positions of the axes and the markers (black points) are based on the actual group mean data from this study, while the generic skeleton object is fitted between the markers, and is not meant to be an actual anatomical representation of the axes relative to the bones of the foot. 

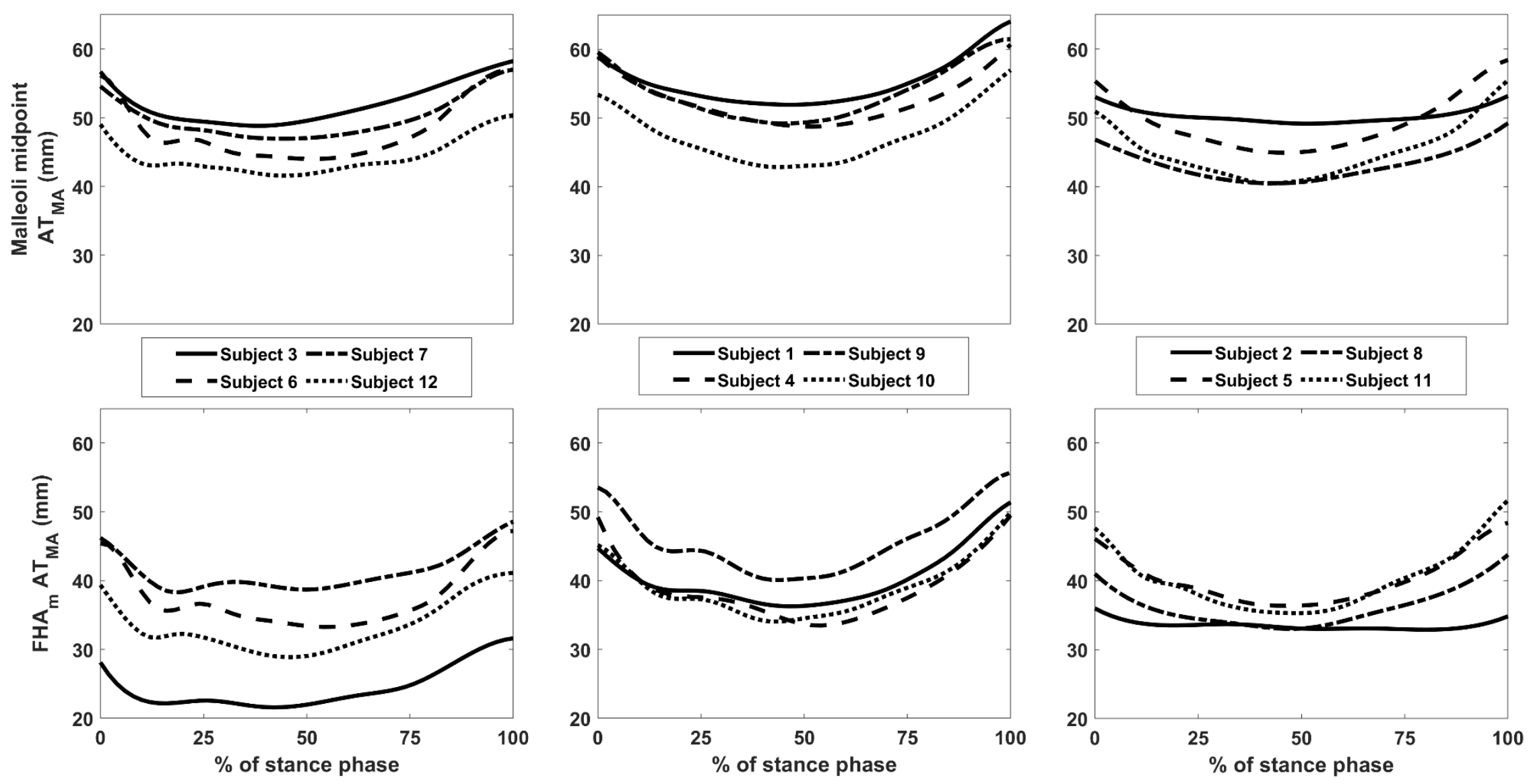

Figure 3. Subject mean $\mathrm{AT}_{\mathrm{MA}}$ curves (mean of subject's trials) across stance phase, split in three panels for ease of visualisation. 


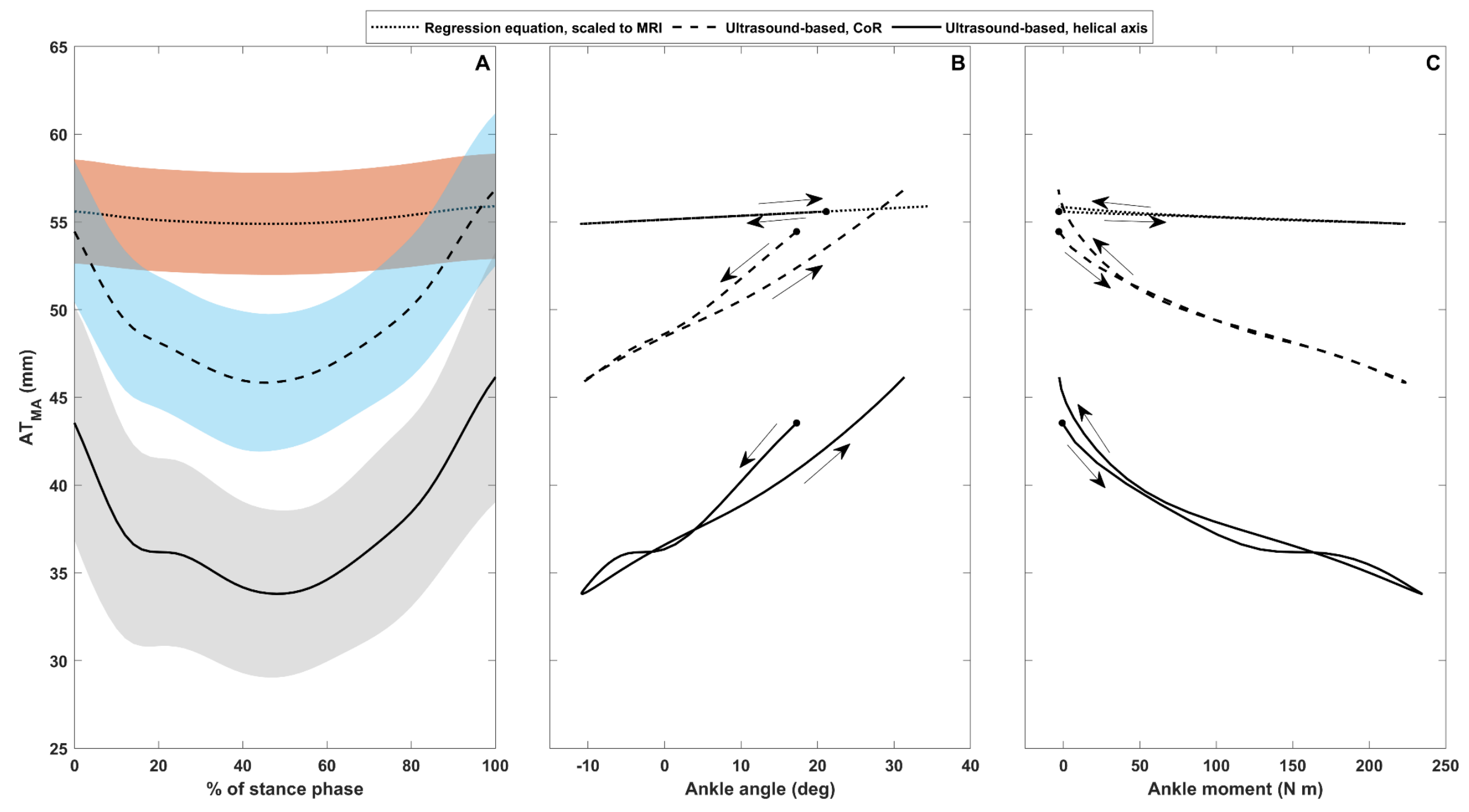

Figure 4. (A) Group mean curves of $\mathrm{AT}_{\mathrm{MA}}$ across stance phase. (B) Group mean curves of $\mathrm{AT}_{\mathrm{MA}}$ vs. dorsi-/ plantar-flexion angle (-/+) during stance phase $\left(0^{\circ}\right.$ corresponds to a $90^{\circ}$ angle between the long axis of the tibia and the foot sole). (C) Group mean curves of AT $\mathrm{MA}$ vs. ankle dorsi-/ plantar-flexion moment (-/+) during stance phase. Shaded areas on (A) represent the SD around the mean curve. Black markers on curves in graphs B and C indicate 


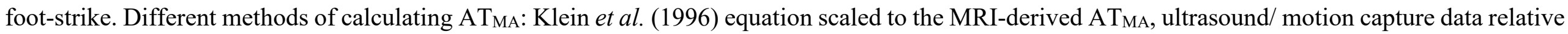

to the malleoli midpoint (CoR), and ultrasound/ motion capture data relative to the ankle $\mathrm{FHA}_{\mathrm{m}}$. 

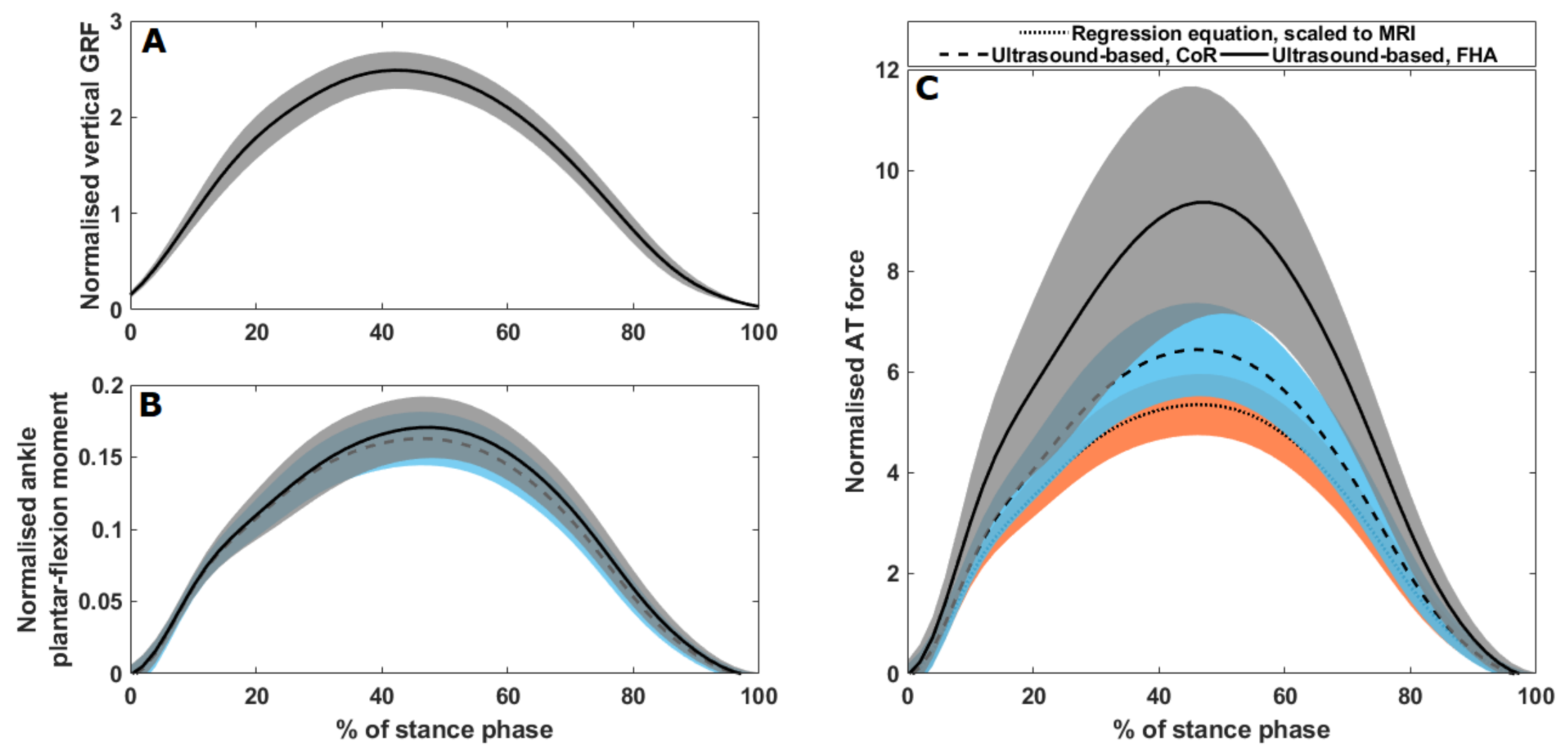

Figure 5. Group mean curves of (A) vertical ground reaction force (normalised to bodyweight), (B) ankle dorsi-flexion (-)/ plantar-flexion (+) moment (normalised to bodyweight times height) calculated relative to the malleoli midpoint (dashed line) and relative to the ankle FHA $($ solid line), and (C) AT force across stance phase (normalised to bodyweight). Shaded areas represent the SD around the mean curve. Different methods of calculating AT $\mathrm{MA}$ presented on (C): Klein et al. (1996) equation scaled to the MRI-derived AT $\mathrm{MA}_{\mathrm{MA}}$, ultrasound/ motion capture data relative to the malleoli midpoint (CoR), and ultrasound/ motion capture data relative to the ankle FHAm. 


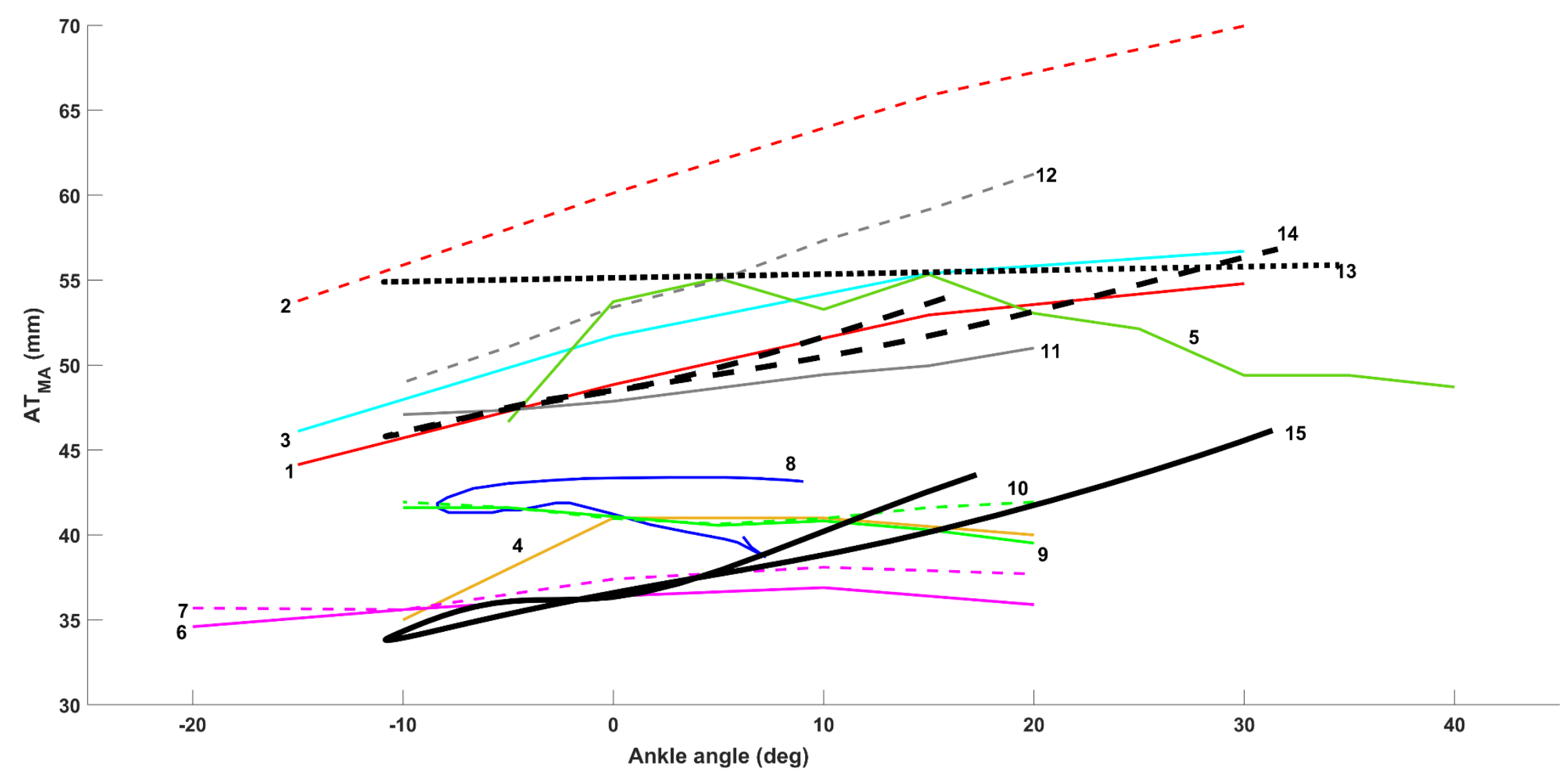

Figure 6. $\mathrm{AT}_{\mathrm{MA}}$ vs. ankle angle from this study (black curves), plotted with previously reported $\mathrm{AT}_{\mathrm{MA}}$ values from the literature. Positive/ negative angles denote ankle plantar-/ dorsi-flexion, with $0^{\circ}$ when the long axis of the tibia is at $90^{\circ}$ with the sole of the foot. Presented data from: $(1-$ solid red) Maganaris (2004) - MRI/ Geometric method at rest, (2 - dashed red) Maganaris (2004) - MRI/ Geometric method during MVC, (3 - light blue) Fath et al. (2010) MRI/ Geometric method at rest, (4 - yellow) Hashizume et al. (2012) - MRI/ FHA at rest, (5 - dark green) Sheenan (2012) - MRI/ FHA during a loaded condition, (6 - solid purple) Manal et al. (2013) - Ultrasound with motion capture/ malleoli midpoint at rest, (7 - dashed purple) Manal et al. (2013) - 
Ultrasound with motion capture/ malleoli midpoint during a MVC, (8 - light green) Rasske et al. (2017) - Ultrasound with motion capture/ malleoli midpoint during walking, (9 - light green solid) Wade et al. (2019) - Ultrasound with motion capture/ malleoli midpoint during an unloaded condition, (10 - light green dashed) Wade et al. (2019) - Ultrasound with motion capture/ malleoli midpoint during a loaded condition, (11 - grey solid) Wade et al. (2019) - Ultrasound with motion capture/ FHA during an unloaded condition, (12 - grey dashed) Wade et al. (2019) - Ultrasound with motion capture/ FHA during a loaded condition, (13 - black dotted) Current study - Regression equation. scaled to MRI, (14 - black dashed) Current study - Ultrasound with motion capture/ malleoli midpoint during running, and (15 - black solid) Current study - Ultrasound with motion capture/ FHA during running. 